\title{
Stabilizing the spatial position of a quadrotor by the backstepping procedure
}

\author{
Dmytro Kucherov $^{1}$, Andrei Kozub ${ }^{2}$, Olha Sushchenko ${ }^{3}$, Ruslan Skrynkovskyy ${ }^{4}$ \\ ${ }^{1}$ Department of Computerized Control Systems, National Aviation University, Ukraine \\ ${ }^{2}$ National Space Facilities Control and Test Center, State Space Agency, Ukraine \\ ${ }^{3}$ Department of Aerospace Control Systems, National Aviation University, Ukraine \\ ${ }^{4}$ Department of Business Economy and Information Technology, Lviv University of Business and Law, Ukraine
}

\begin{tabular}{l}
\hline \hline Article Info \\
\hline Article history: \\
Received May 7, 2021 \\
Revised Jul 7, 2021 \\
Accepted Jul 14, 2021 \\
\hline
\end{tabular}

Keywords:

Backstepping procedure

Control system

Quadrotor

Spatial position

Stabilizing

\begin{abstract}
The paper considers the problem of synthesizing a control law that stabilizes the spatial position of an airborne object. The control object is a quadrotor with nonlinear dynamics. To solve the stabilization problem, a mathematical model of the quadrotor has been developed, taking into account its position in the Cartesian and Euler coordinate systems. The new control law has been synthesized using the backstepping procedure. This control law is based on the Lyapunov-type stabilization criterion. New results analysis of the quadrotor dynamics, where has been showing the dependence of the control accuracy on the parameter of the stabilization criterion also presented. An algorithm for the directed search of the procedure parameter also has been proposed. It ensures the desired quality of the transient process. Simulation results confirming the results of theoretical research have been presented as well.
\end{abstract}

This is an open access article under the CC BY-SA license.

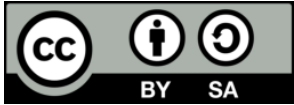

\section{Corresponding Author:}

Dmytro Kucherov

Department of Computerized Control Systems

National Aviation University

1, Liubomyra Huzara ave, Kyiv, 03058, Ukraine

Email: d_kucherov@ukr.net

\section{INTRODUCTION}

Despite the growing number of applications for quadrotors, the problem of building an effective control system for stabilizing the spatial position of this device still exists. Stable flight, the accuracy of maneuvering, and the performance of the flight task as a whole depend on the quality of the control system. The designers of quadcopter control systems need to take into account the nonlinear dynamics of the controlled vehicle associated with the parametric uncertainty of the dynamic plant, possible variants of unmodelled dynamics, as well as the influence of external flight factors, including wind and obstacles on the route, that in general requires effective maneuvering [1].

The control system of the UAV, as a rule, relies on the dynamics of second-order models, characterized by weak stability, and the feedback principle, which does not allow solving flight problems completely. The control system designer should also take into consideration the limited space available to accommodate airborne avionics and batteries. Modern miniature electronic devices allow the implementation of complex control algorithms that can guarantee the safety of all phases of quadrotor flight. Hence, it is necessary to search for the appropriate algorithms for controlling the dynamics of a quadrotor, the effectiveness of which is confirmed by adequate mathematical modeling. 
The nonlinearity of the quadrotor dynamics is explained mainly by the imaginary roots of the characteristic equation obtained based on its mathematical model, which determines the complexity of the control law synthesis [2]. Increasing the dimension of a dynamical system leads to a significant increase in the computational complexity of the control law; besides, it needs additional constraints when setting the problem.

The general approach to build mathematical models of designed UAV control systems can found in [3] and in the references therein. As we have known that the flight dynamic of aircraft of the type under consideration is substantially nonlinear since there is a mutual dependence of the longitudinal and transverse motions. Also, this aircraft is sensitive to external disturbances, and it has some uncertainty regarding the parameters of the controlled plant that cannot be ruled out.

The authors [4]-[6] utilized a control system scheme is implementing the inverse dynamics of a controlled object using a PID (PD) controller. As is known [7], [8], the PID controller requires adjustment; the high-frequency components of the error signal introduce additional noise into the control channel; also, large-amplitude control pulses may appear in cases of a jump change in the error signal. The identification of a mathematical model of a fixed-wing UAV is proposing in [9]. In this case, the estimates of the unknown coefficients of the transfer functions were found by the least-squares method; and the sought variables are determining by the dynamics of the acting system. It assumes an optimal control system in the system has.

Wang and $\mathrm{Li}$ [10] propose a three-dimensional visualization of a UAV with four tilt rotors. A control system includes blocks of a tracking differentiator, an extended state observer, and nonlinear error feedback. However, there is no information regarding the quality of the constructed regulator there. The control system, where the ratio controller is applied based on the reverse step algorithm and the desired function of angular accelerations, is used in a micro-machine like a bat, is presented in [11]. The gradient (steepest descent) approach is used in the control system of UAV [12]-[14]. It is based on the integral differential model of the control object, which is developed by the authors and used in the decision support system for the apparatus control.

It should be noted that the central place of leading conferences and journal publications on control theory (see, for example, [15]-[17]) has recently been devoted to a review of control methods, which also include the backstepping procedure. So, the backstepping technique history for solving partial differential equations has been presented by the authors [15]. Comparison of three control methods for nonlinear objects, namely: tracking mode, back step method, and linearized feedback, according to publications covering a large time interval, is presented in [16]. The authors give preference to the first two management methods, rightly pointing out their existing shortcomings that have taken place in practice. Unlike the previous publication, in addition to those considered, control based on fuzzy logic is studied by Nasr et al. [17]. Unfortunately, preference for one method or another should be given only after an accurate study of the control object and its operating conditions.

It should be noted, the parameters of the transient process using the backstepping algorithm are not always satisfactory, and it requires a new solution search. So, the authors [18], [19] were encouraged to find the best parameters of the backstepping controller by particle swarm optimization. In the paper [19], the optimal parameters of the backstepping algorithm are found by the method of an artificial bee colony, which makes it possible to obtain a controlled process better than with their arbitrary choice. However, the proposed computational scheme is time-consuming. Recent publications on the applications of the backstepping procedure for providing satellite navigation and control of the UAV network have been presented in papers [20]-[23], respectively. Therefore, the use of advanced control methods is in the interest of future use such as space and UAV network control.

The backstepping procedure has recently become more popular for electrical machine control [24]-[28]. The peculiarity of the backstepping algorithm in this works is increasing the Lyapunov function candidate on two to five derivatives. Unfortunately, the procedure parameters are chosen most often heuristically or by trials and errors method search. Similar approach authors [29] used for control of DC-DC buck converter.

In works [30]-[32], a photovoltaic cell is used in a control loop to solve various control problems. The practical application of the reverse step procedure does not differ from the method mentioned above. In paper [32], as adaptive backward step procedure is understanding the construction of a candidate Lyapunov function with an additional term.

An analysis of the above publications allows us to conclude that a controller based on a backstepping algorithm for a quadrotor is of interest to researchers. However, there is a problem of choosing sufficient controller parameters, which is currently being solving by trial and error. The difference between a quadrotor and other types of control objects is the need for spatial control. In this case, a dynamic plant is describing by a large number of coordinates: spatial coordinates and their speeds and angular coordinates and their speeds, and the stabilization of its spatial position is providing by fourth motors. This work is the development of our idea proposed in [33], [34], and in the current project, we study the parameter of the 
backstepping procedure for typical quadrotor moves. A new algorithm for setting the parameters of the backstepping procedure, depending on the desired quality of control, is proposed, and it is our contribution to the paper. The paper also provides a theoretical basis for the algorithm and analysis of the simulation results.

\section{SYSTEM MODEL}

The quadrotor diagram, coordinate systems, and the forces acting on the control object shown in Figure 1, where we introduce the notation $X Y Z$ ( $O$-frame) is a fixed coordinate system connected by the Earth, $X^{\prime} Y^{\prime} Z^{\prime}$ (UAV-frame) is a moving coordinate system, its origin coincides with the UAV's center of gravity, variables $\varphi, \theta, \psi$ correspond to the angles of roll, pitch, and yaw in the Earth coordinate system, where $\varphi, \theta$ are in the interval $]-\pi / 2 ; \pi / 2[$, a $\psi \in]-\pi ; \pi[$.

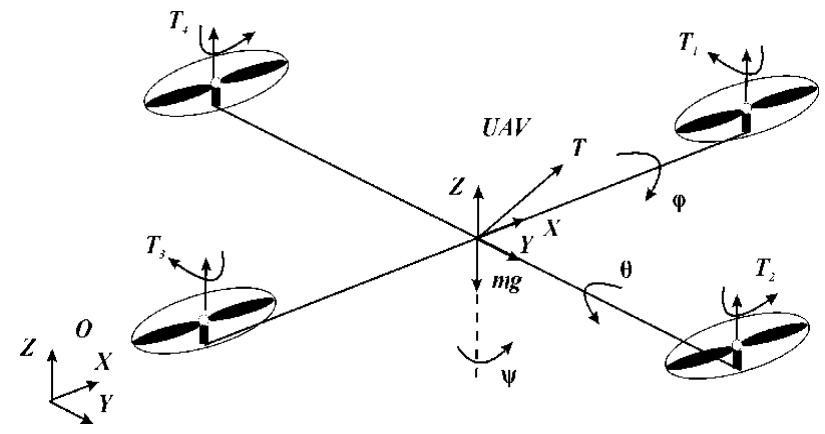

Figure 1. Scheme of forces acting on the quadrotor in the $X^{\prime} Y^{\prime} Z^{\prime}$ coordinate system

The equations of the dynamics of the quadrotor in 3D space are written in the form

$$
\left|\begin{array}{cc}
m I & 0 \\
0 & J
\end{array}\right|\left|\begin{array}{c}
\dot{V} \\
\dot{\Omega}
\end{array}\right|+\left|\begin{array}{c}
\Omega \times m V \\
\Omega \times J \Omega
\end{array}\right|=\left|\begin{array}{c}
\mathfrak{I} \\
\mathbb{N}
\end{array}\right|
$$

where $V=\left(V_{x}, V_{y}, V_{z}\right)^{T}=(\dot{x}, \dot{y}, \dot{z})^{T}$ is the vector of linear, and $\Omega=\left(\Omega_{\phi}, \Omega_{\theta}, \Omega_{\psi}\right)^{T}=(\dot{\phi}, \dot{\theta}, \dot{\psi})^{T}$ is the vector of angular velocities, $m$ is the mass of the vehicle, $J \in \mathfrak{R}^{3 \times 3}$ is the inertia matrix of the control object, $I$ is the identity matrix, $\mathfrak{I}$ is the resultant force, $\mathfrak{\aleph}$ is the resultant moment acting on the center of mass of the UAV.

Neglecting small values of external forces, the resulting force determining the movement of the $\mathrm{UAV}$ in the vertical plane is written as

$$
\mathfrak{J}^{T}=\left(\begin{array}{lll}
0 & 0 & u
\end{array}\right)
$$

In the Eulerian coordinate system, the UAV orientation relative to the inertial coordinate system $X Y Z$ can be represented by rotation matrix $R$

$$
\mathfrak{I}_{V}=R(\psi, \theta, \phi) \mathfrak{I}
$$

where

$$
R=\left(\begin{array}{ccc}
C_{\psi} C_{\theta} & C_{\psi} S_{\theta} S_{\phi}-S_{\psi} C_{\phi} & C_{\psi} S_{\theta} C_{\phi}+S_{\psi} S_{\phi} \\
S_{\psi} C_{\theta} & S_{\psi} S_{\theta} S_{\phi}+C_{\psi} C_{\phi} & S_{\psi} S_{\theta} C_{\phi}-C_{\psi} S_{\phi} \\
-S_{\theta} & C_{\theta} S_{\phi} & C_{\theta} C_{\phi}
\end{array}\right)
$$

The matrix (4) uses notations $C_{\alpha}=\cos (\alpha), S_{\alpha}=\sin (\alpha)$. Taken into consideration (3) and (4) we can rewrite formula (1) in the following form

$$
\begin{aligned}
& m \ddot{x}=\bar{u}_{1}\left(C_{\psi} S_{\theta} C_{\phi}+S_{\psi} S_{\phi}\right) \\
& m \ddot{y}=\bar{u}_{1}\left(S_{\psi} S_{\theta} C_{\phi}+C_{\psi} S_{\phi}\right) \\
& m \ddot{z}=\bar{u}_{1} C_{\theta} C_{\phi}-m g
\end{aligned}
$$


Let $J_{1}, J_{2}, J_{3}$ are moments of quadrotor inertia for axes $X, Y$ and $Z$ passing through its center of mass, respectively. $\dot{\phi}, \dot{\theta}, \dot{\psi}$ are angular rates regarding axes $X, Y$ and $Z$. As it is known [2], [23], that if external forces are absent, the differential equations of angular rates can be represented in the form

$$
\begin{aligned}
& J_{1} \ddot{\phi}(t)=\left(J_{2}-J_{3}\right) \dot{\theta}(t) \dot{\psi}(t) \\
& J_{2} \ddot{\theta}(t)=\left(J_{3}-J_{1}\right) \dot{\psi}(t) \dot{\phi}(t) \\
& J_{3} \ddot{\psi}(t)=\left(J_{1}-J_{2}\right) \dot{\phi}(t) \dot{\theta}(t)
\end{aligned}
$$

The (8)-(10) are Euler's dynamic equations. When taking into account the action of engines $M_{1}-M_{4}$, (8)-(10) look like

$$
\begin{aligned}
& J_{1} \ddot{\phi}(t)=\left(J_{2}-J_{3}\right) \dot{\theta}(t) \dot{\psi}(t)-J_{r} \dot{\theta}(t) \Omega+d \bar{u}_{3}(t) \\
& J_{2} \ddot{\theta}(t)=\left(J_{3}-J_{1}\right) \dot{\psi}(t) \dot{\phi}(t)+J_{r} \dot{\phi}(t) \Omega+d \bar{u}_{4}(t) \\
& J_{3} \ddot{\psi}(t)=\left(J_{1}-J_{2}\right) \dot{\phi}(t) \dot{\theta}(t)+\bar{u}_{2}(t)
\end{aligned}
$$

In formulas $(11)-(13) d$ is the distance between the center of mass of UAV and engine with $\mathfrak{J}_{i}, J_{r}$ is the rotor inertia, $\bar{u}_{1}, \bar{u}_{2}, \bar{u}_{3}, \bar{u}_{4}$ are mechanical forces providing the rotational motion of the drone, are represented by the equations

$$
\begin{aligned}
& \bar{u}_{1}=k_{1} \Omega_{1}^{2}+k_{2} \Omega_{2}^{2}+k_{3} \Omega_{3}^{2}+k_{4} \Omega_{4}^{2} \\
& \bar{u}_{2}=k_{1} \Omega_{1}^{2}+k_{2} \Omega_{2}^{2}-k_{3} \Omega_{3}^{2}-k_{4} \Omega_{4}^{2} \\
& \bar{u}_{3}=k_{4} \Omega_{4}^{2}-k_{2} \Omega_{2}^{2} \\
& \bar{u}_{4}=k_{1} \Omega_{1}^{2}-k_{3} \Omega_{3}^{2}
\end{aligned}
$$

$k_{i}$ is a factor connecting the moment with the angular velocity $\Omega_{i}$ of the $i$ th motor, in (14)-(17). Take into consideration the identity of the motors, the symmetry of their location, and the rigidity of the structure, and we can establish that at a fixed height (Z-axis) the moments $J_{1}$ and $J_{2}$ in (11)-(13) are equal, i.e.

$$
J_{1}=J_{2}=J
$$

The rotational dynamics of the quadrotor by (11)-(13) can rewrite in the following form

$$
\begin{aligned}
& \ddot{\phi}(t)=\frac{1}{J}\left[\left(J-J_{3}\right) \dot{\theta}(t) \dot{\psi}(t)-J_{r} \dot{\theta}(t) \Omega+d \bar{u}_{3}(t)\right] \\
& \ddot{\theta}(t)=\frac{1}{J}\left[-\left(J-J_{3}\right) \dot{\psi}(t) \dot{\phi}(t)+J_{r} \dot{\phi}(t) \Omega+d \bar{u}_{4}(t)\right] \\
& \ddot{\psi}(t)=\frac{1}{J} \bar{u}_{2}(t)
\end{aligned}
$$

Given the initial state $\psi(0)$ and knowing the moment $u_{3}$, the coordinate $\psi(\mathrm{t})$ can be found by double differentiation (21)

$$
\psi(t)=\psi(0)+\frac{1}{J} \bar{u}_{2} d t^{2}
$$

that it follows from the independence of the coordinates $\varphi(t), \theta(t)$.

The stabilization of the coordinate $\psi$ is possible if the angular velocities take zero values, and the equality $\bar{u}_{2}=$ const is ensured, where $\psi(t)=\Psi=$ const. We introduce restrictions on the moments $\bar{u}_{3}, \bar{u}_{4}$

$$
\left|u_{3}(t)\right| \leq \bar{u}_{3}=U,\left|u_{4}(t)\right| \leq \bar{u}_{4}=U
$$


We also introduce a new notation

$$
\omega=\frac{J-J_{3}}{J} \dot{\psi}, \Phi=\dot{\phi}, \Theta=\dot{\theta}, l=\frac{d}{J}
$$

and after substitution of expressions (23) into (20), (21), we obtain

$$
\begin{aligned}
& \dot{\Phi}=\omega \Theta-\frac{J_{r}}{J} \Theta \Omega+l \bar{u}_{3}=\omega^{\prime} \Theta+l \bar{u}_{3} \\
& \dot{\Theta}=-\omega \Phi+\frac{J_{r}}{J} \Phi \Omega+l \bar{u}_{4}=-\omega^{\prime} \Phi+l \bar{u}_{4}
\end{aligned}
$$

A new variable $\omega^{\prime}=\omega-\frac{J_{r}}{J} \Omega$ is introduced in (23), (24). The non-linearity of this model has mainly defined constraints of elements and their many inputs that can be a step or harmonic behavior. As accomplish [2] system (23), (24) has imaginary roots of their characteristic equation and because it has nonlinear dynamics. Now our goal is to synthesize a control law that will provide $e_{\Phi} \rightarrow 0, e_{\Theta} \rightarrow 0$, for $t \rightarrow \infty$ without noise in canals measurement of coordinates of UAV; $e_{\Phi}, e_{\Theta}$ are the errors of control by angles.

\section{THE SYNTHESIS OF CONTROL LAW}

The control plant represented by (23), (24) has nonlinear dynamics and can be described by the transfer function of a conservative second-order element with two imaginary poles.

Consider the case when $\bar{u}_{3}=$ const and without loss generality we assume $\bar{u}_{3}=0$. The system of (23), (24) by multiplying on $(l \omega)^{-1}$ the equation (23) and on coefficient $l^{-1}$ the equation (24) we converting these equations to the equivalent form

$$
\begin{aligned}
& \dot{\alpha}=\beta \\
& \dot{\beta}=-\left(\omega^{\prime}\right)^{2} \alpha+\bar{u}_{4}
\end{aligned}
$$

Here, the expressions $\alpha=(l \omega)^{-1} \Phi$ and $\beta=l^{-1} \Theta$ are used. Next, following [33], a new variable $\beta=\kappa(\alpha)$ is introduced. We assume that the system described by (25), (26) can be stabilized by state feedback using the control variable $\beta=\kappa(\alpha)$, where $\kappa(0)=0$. Then, if the variable $\kappa(\alpha)$ is found, we will consider the solution systems with $\dot{\alpha}=\kappa(\alpha)$ an asymptotically stable equation.

Here we also introduce a candidate of Lyapunov function satisfying the inequality

$$
\dot{L}_{1}(\alpha)=\frac{\partial L}{\partial \alpha} \dot{\alpha}(\alpha, \beta) \leq-W(\alpha), \alpha, \beta \in \Re
$$

where $W(\alpha)$ is a positive definite function.

Let $e=\beta-\kappa(\alpha)$, then (25), (26) can be rewritten as

$$
\begin{aligned}
& \dot{\alpha}=\kappa(\alpha)+e \\
& \dot{e}=-\left(\omega^{\prime}\right)^{2} \alpha+\bar{u}_{4}-\dot{\kappa}(\alpha)=v
\end{aligned}
$$

where $v$ is a pseudo-control variable that stabilizes the variable $\alpha$. Following the methodology [33], we also introduce a candidate Lyapunov function for (28), (29) and we obtain

$$
\dot{L}_{2}=\frac{\partial L}{\partial \alpha} \kappa(\alpha)+e v \leq-W(\alpha)+e v
$$

By choosing

$$
v=-\lambda e,
$$

we get

$$
\dot{L}_{2} \leq-W(\alpha)-\lambda e^{2}
$$

Here $\lambda$ is the parameter of speed convergence of the control process. 
You can see that the origin corresponding to the point $\alpha=0, e=0$ is asymptotically stable. If $\kappa(0)=0$, it can be established that the starting point with coordinates $\alpha=0, \beta=0$ is also stable. Applying approach [33] and expressions for $v, e, \dot{\kappa}(\alpha)$, one can obtain the state feedback law

$$
\bar{u}_{4}=\left(\omega^{\prime}\right)^{2} \alpha+\frac{\partial \kappa(\alpha)}{\partial \alpha} \beta-W(\alpha)-\lambda(\beta-\kappa(\alpha))
$$

The diagram for realizing this algorithm is presented in Figure 2. Figure 2 shows that to implement control, the backstepping controller only needs information about the values $\alpha, \beta$.

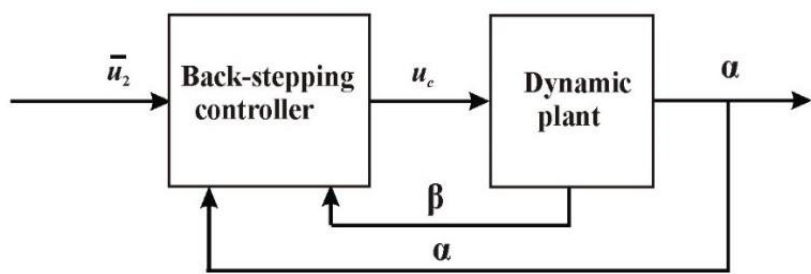

Figure 2. Diagram of the algorithm (34)

The considered approach proved the next theorem.

Theorem. Let dynamic plant described by equations system (25), (26), then control law (33) is stabilizing for equations system (25), (26).

Proposition. Let $\Omega_{i}, \Omega^{*}$ are measured and reference vectors of angular velocities, respectively, $1 \leq i \leq N ; N$ is the number of measurements made; $\varepsilon\left(\lambda_{i}\right)$ is measured and permissible root-mean-square values of measurement errors that are given the expression

$$
\varepsilon\left(\lambda_{i}\right)=\sqrt{\frac{1}{N} \sum_{i=1}^{N}\left|\Omega\left(\lambda_{i}\right)-\Omega *\right|^{2}}
$$

Then there is value $\lambda_{\max } \leq \Lambda$ ( $\Lambda$ is the stability boundary), which manifests $\varepsilon_{e l}=\varepsilon\left(\lambda_{\max }\right)$ and satisfies (33), (34) that it is

$$
\lambda \arg \min _{\substack{\lambda_{i}, 1 \leq i \leq N} \quad \sqrt{\varepsilon\left(\lambda_{i}\right)}} \max
$$

Formulae (34), (35) establishes a procedure for choosing a sufficient value of $\lambda$ for dynamic plant (25), (26).

The control system that ensures tracking by quadrotor of varying input for the proposed mathematical model is shown in Figure 3. Due to improving tracking accuracy, the control system builds on the principle of feedback [35]. We also assume that the quadrotor has sensors that allow obtaining linear $x, y, z$, and angular $\varphi, \theta, \psi$ coordinates of the quadrotor's position in space without noises, used by controllers to generate control signals.

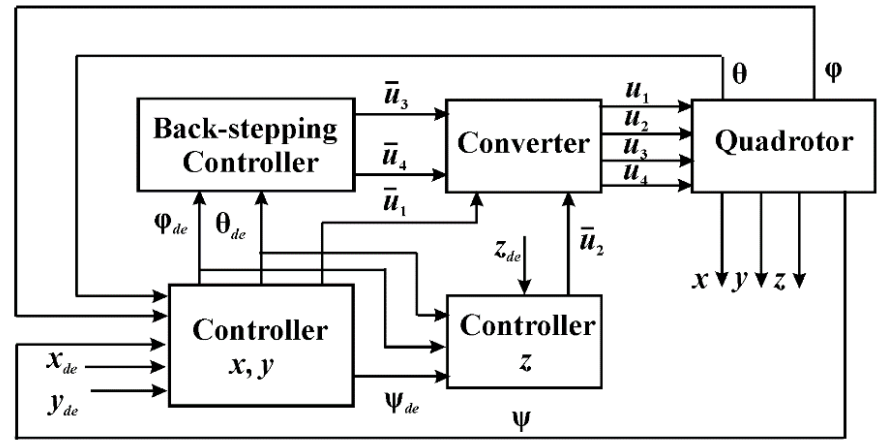

Figure 3. The structure of the control system for quadrotor 
There are three controllers in the control system. Controller $x, y$ generates a control signal $\bar{u}_{1}$, which creates forces that ensure movement quadrotor in the $x, y$ plane. This controller functions according to the mathematical model (5), (6). The controller $z$ generates a control signal $\bar{u}_{2}$, which affects the movement plant along the third coordinate, its movement given by the (7), (21). The backstepping controller sets the angular positions $\varphi, \theta$ of the quadrotor when moving along a given trajectory, generating control signals $\bar{u}_{3}, \bar{u}_{4}$. This controller functions according to the mathematical model (23), (24), (33). The converter transforms control signals into forces (14)-(17) that determine the quadrotor position in space at any one time.

\section{RESULTS AND DISCUSSION}

As follows from the mathematical model of the quadrotor described by (25)-(27), the angular dynamics represented by (23), (24) do not depend on the spatial coordinates of the UAV. At the same time, the spatial coordinates (5)-(7) are completely determined by the angular coordinates and their derivatives in time. Thus, the general control system consists of two independent control subsystems, the one controls the translational dynamics, its output parameters are the initial data for the second subsystem.

The orientation of the quadrotor in three-dimensional space depends on the control of the angular coordinates. To implement hovering and stabilize its position for some time, the angular rates of roll, pitch and yaw tend to be kept equal to zero values. Here it is suggested to use the backstepping procedure for this task. The initial parameters for simulating the dynamics of the prototype quadrotor are given in Table 1 .

Table 1 . Some physical parameters of the quadrotor prototype used in the simulation

\begin{tabular}{clcc}
\hline Parameter & \multicolumn{1}{c}{ Description } & Value & Unit \\
\hline$m$ & mass of the vehicle & 0.624 & $\mathrm{Kg}$ \\
$d$ & $\begin{array}{l}\text { distance between the center } \\
\text { of mass of UAV and engine }\end{array}$ & 0.3 & $\mathrm{M}$ \\
& moment inertia for axes $X$ & 0.0015 & $\mathrm{~kg} \cdot \mathrm{m}^{2}$ \\
$J_{1}$ & moment inertia for axes $Y$ & 0.0015 & $\mathrm{~kg} \cdot \mathrm{m}^{2}$ \\
$J_{2}$ & moment inertia for axes $Z$ & 0.0027 & $\mathrm{~kg} \cdot \mathrm{m}^{2}$ \\
$J_{3}$ & rotor inertia & $6.8 \cdot 10^{-5}$ & $\mathrm{~kg} \cdot \mathrm{m}^{2}$ \\
$J_{\mathrm{r}}$ & & &
\end{tabular}

Further, we will study the three variants of action of the control system: two cases only backstepping controller in which we show its reaction on the step and harmonic functions, and full action control for quadrotor motion in $X Y$-plane.

\subsection{Case 1 study: input is a step function}

Modeling of the control law (33) for the dynamic system (25), (26) with the parameter $\omega^{\prime}=42 \mathrm{rad} / \mathrm{s}$ on the interval $T=10 \mathrm{~s}$ has been performed in the Matlab environment.

The unit step function $\bar{u}_{4}=1(t)$ fed to the input of the dynamic system; the system response without stabilization is shown in Figure 4, the stabilization of the system with the parameters of the control law (33) $\lambda=2,20$ shown in Figure 5(a) and (b). In this Figure, the dotted line shows the system output when stabilized by local feedback. Figure 6 show the function $\varepsilon(\lambda)$. This function is useful for the procedure (34), (35) assessment.

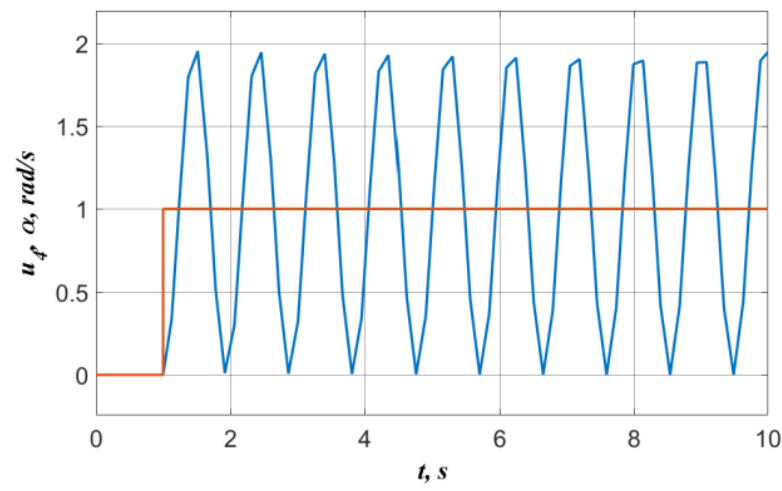

Figure 4. Functions of $\bar{u}_{4}(t)$ (red) and $\alpha(t)$ (blue) without stabilization 


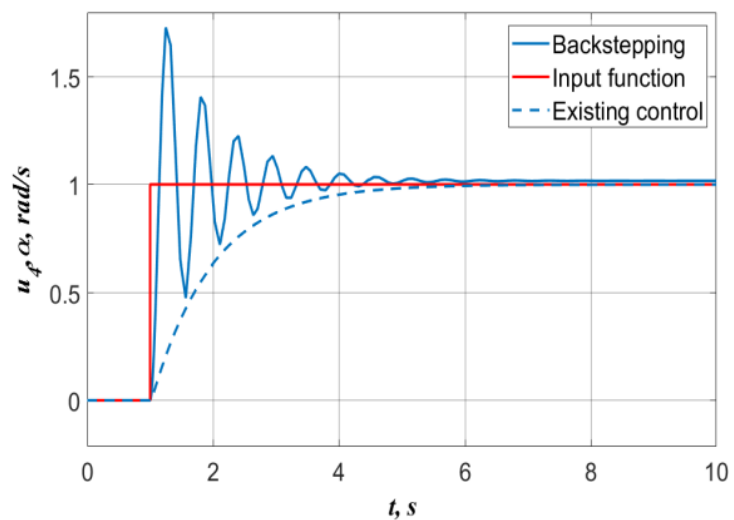

(a)

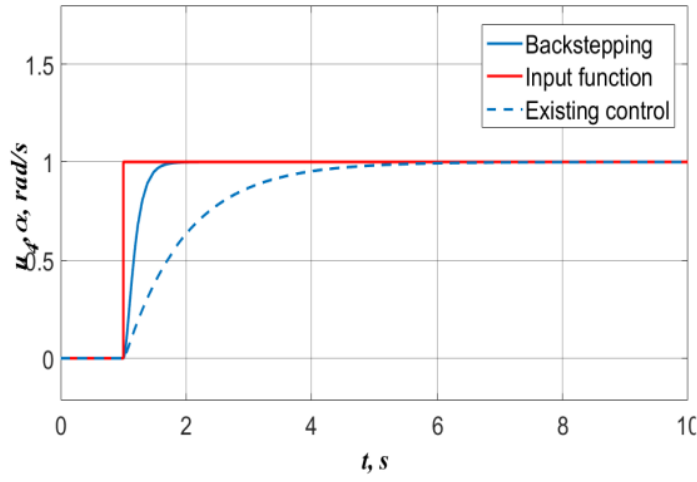

(b)

Figure 5. Functions of $\bar{u}_{4}(t)$ (red) and $\alpha(t)$ (blue) after stabilization; (a) $\lambda=2$ and (b) $\lambda=20$

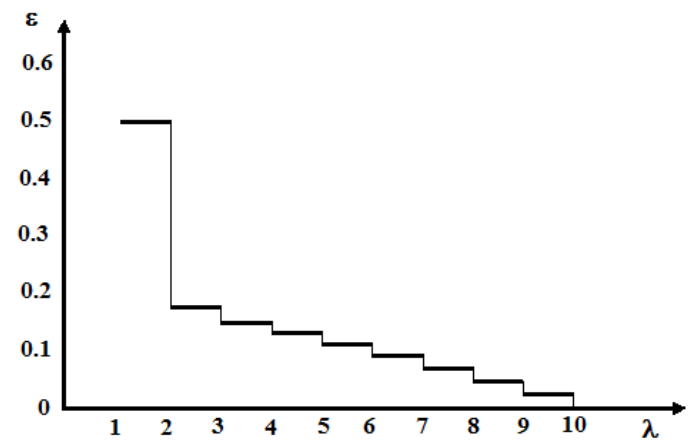

Figure 6. Function $\varepsilon(\lambda)$

From this graph, we can note dependence $\varepsilon(\lambda)$ monotone decreasing, and adequate error level can realize for $\lambda \geq 7$ in given parameters dynamic plant.

\subsection{Case 2 study: input is a harmonic function}

A similar Case 1 this study was carried out for the case when the input changes according to the harmonic function with $\omega=0.16 \mathrm{rad} / \mathrm{s}$, i.e. $\bar{u}_{4}(t)=\sin \omega \mathrm{t}$, studies for a signal with duration $T=10 \mathrm{~s}$ are shown in Figures 7, 8(a) and (b). The dotted line in the last figure is used to show the result of the local feedback stabilization.

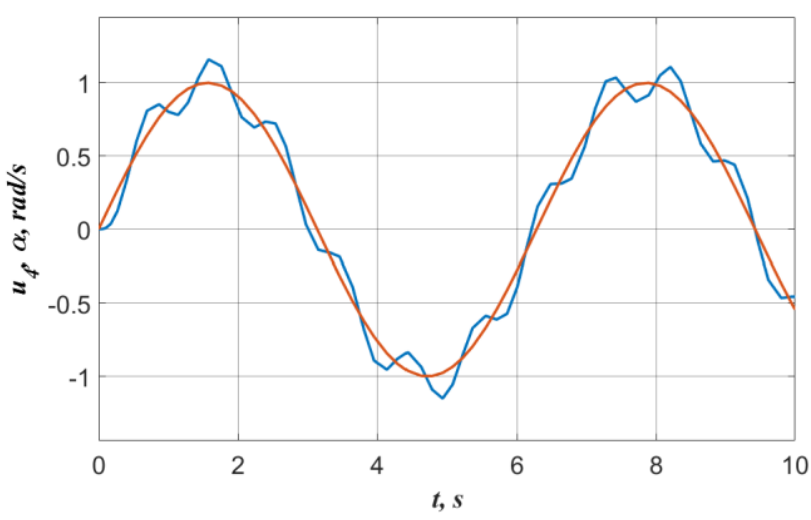

Figure 7. Functions of $\bar{u}_{4}(t)=\sin \omega t$ (red) and $\alpha(t)$ (blue) before stabilization 


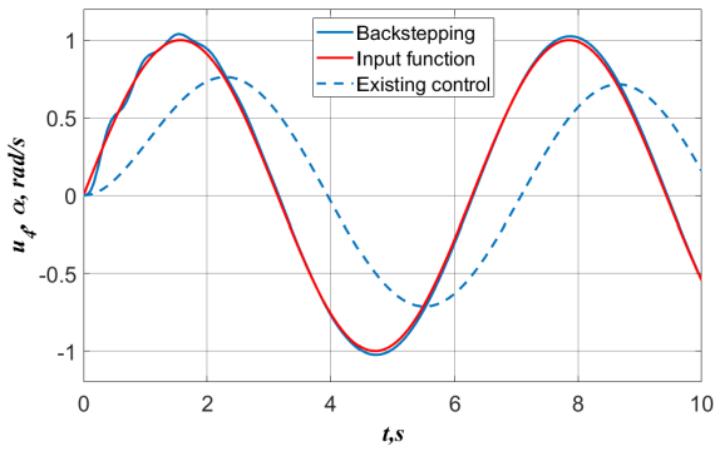

(a)

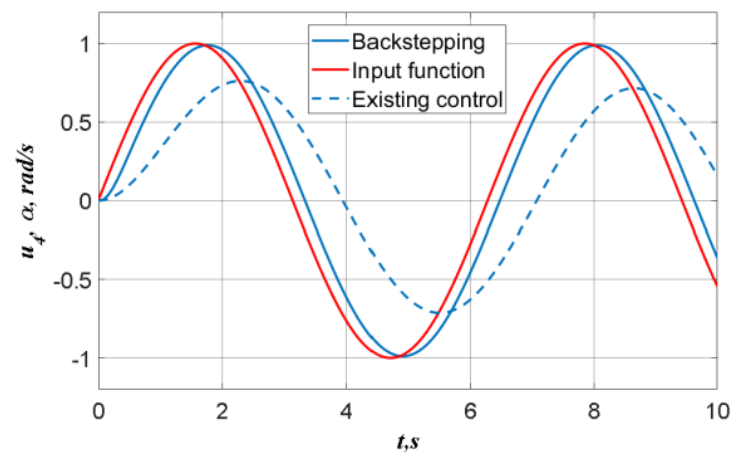

(b)

Figure 8. Functions of $\bar{u}_{4}(t)=\sin \omega t$ (red) and $\alpha(t)$ (blue) after stabilization; (a) $\lambda=2$ and (b) $\lambda=20$

As you can see from Figure 8(b) the control system has constant error for this type of input signal. Local feedback stabilization leads to distortion of the input signal waveform.

\subsection{Case 3 study: spatial stabilization}

We also propose a general scheme of the analysis of dynamics in 3D space. It is necessary for the completeness of the study of the mathematical model, which takes into account the mutual influence of all four moments of the quadrotor on the trajectory of motion.

The diagram contains additional designations $x_{d e}, y_{d e}, z_{d e}, \varphi_{d e}, \theta_{d e}, \psi_{d e}$, which are the outputs of the comparison elements of the corresponding coordinates. The input signal of the control system is described by [36]

$$
\left(x-x_{0}\right)^{2}+\left(y-y_{0}\right)^{2}=R^{2}, z=z_{0} .
$$

In this case, the task was to study the controller for tracking a circular path. Initial data for modeling $x_{0}=0 \mathrm{~m}, y_{0}=0 \mathrm{~m}, z_{0}=1 \mathrm{~m}, R=1 \mathrm{~m}, \lambda=10$ and $\varepsilon_{e t}=0.001$.

The result of this simulation is shown in Figure 9; the red curve is the input signal; the blue curve is the UAV trajectory with the existing control; the green curve is the backstepping control.

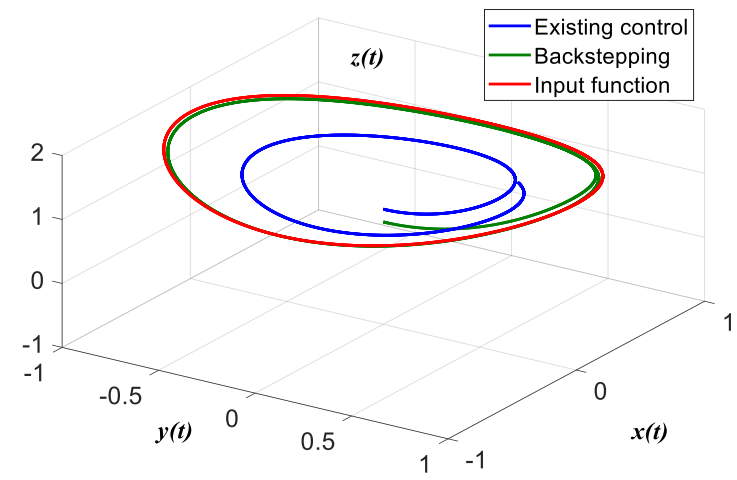

Figure 9. System's dynamic in the 3D space

The results in Figure 9 confirm the effectiveness of the stabilization of systems with nonlinear dynamics. Analysis of Figures 7-9 matches with the main theoretical result regarding the stabilization of a dynamic system with nonlinear dynamics. The quality of control depends significantly on parameter $\lambda$. The analysis of the control law (33), despite some complexity of the analytical inference, shows a relatively simple technical implementation, since the implementation relies on signals present in the system, forming a special type of feedback.

As can be seen from the figures, the regulator using control law (33) compensates for the initial error, stabilizes the mismatch of the roll, pitch, and yaw angular velocities, and maintains their values equal 
to zero. Application of the procedure (35) does it possible to obtain $\lambda=10$ at $\varepsilon_{e t}=0.001$. This investigation does not include noises in the canals of measurements of coordinates. We assume that take into consideration the influence of noises leads to little worsening of accuracy control but this problem requires consideration.

\subsection{Problems for implementation}

The obvious advantage of the proposed control law is the simplicity of technical implementation on a modern element base, the absence of the need to use PID controllers, as well as the problems associated with their tuning. The control system can be implemented on processors, for example, the STM32F4 series, which combines high performance and low power consumption. The paper [37] can serve as a reference point in this direction, where the same processor is used in the feedback loop (PIL by author's edition) to provide the control trajectory of the quadrotor for a finite time in a sliding mode. Nevertheless, the implementation requires separate, additional work related to the development of full hardware and software for the provision of flight tasks, including the construction of a radio control channel, as well as equipment that determines the purpose of the UAV, which is a further logic continuation of this work.

\section{CONCLUSION}

The solution to the problem of the control plant stabilization that has nonlinear dynamics presented in this work. As a controlled object whose mathematical model has multiple imaginary roots of the characteristic equation used a quadrotor. Due to solve the problem of stabilization in this work, the back step procedure has been applied. The algorithm has a configurable parameter, which does not contradict previous studies. However, unlike the known approaches, we offer a tuning algorithm that excludes the trial and error method. In the study, we have determined the dependence of the quality of the transition process from one parameter of the stabilization criterion. This parameter defines the speed of stabilization. Our contribution to this study is the establishing a new algorithm of the quadrotor angular position stabilization by backstepping procedure; definition the dependence quality of control the backstepping procedure parameter. Also, we proposed the algorithm for choosing the suitable value of this parameter by procedure-directed search. We will plan to aim further researches at the realization of the proposed algorithm. Besides, to determine the ways for decreasing dynamic error, and also take into consideration sensor noises.

\section{REFERENCES}

[1] S. Ullah, A. Mehmood, Q. Khan, S. Rehman, and J. Iqbal, "Robust Integral Sliding Mode Control Design for Stability Enhancement of Under-actuated Quadcopter," International Journal of Control, Automation and Systems, vol. 18, pp. 1671-1678, 2020, doi: 10.1007/s12555-019-0302-3

[2] D. Kucherov, O. Sushchenko, A. Rasstrygin, S. Zhdanov, and A. Kozub, "Synthesis of the switching control law for a quadrotor autopilot," International Journal of Engineering \& Technology, vol. 7, no. 4, pp. 3065-3069, Nov 2018, doi: 10.14419/ijet.v7i4.16368.

[3] M. Wasim, M. Ullah, and J. Iqbal, "Taxi model of unmanned aerial vehicle: Formulation and simulation," 20181 st Int. Conference on Power, Energy and Smart Grid (ICPESG), 2018, pp. 1-6, doi: 10.1109/ICPESG.2018.8384506.

[4] G. Farid, M. Hongwei, A. H. Baqar and Q. Liwei, "Inverse dynamics based altitude/attitude control and stabilization of a quadrotor UAV," 2017 9th International Conference on Modelling, Identification and Control (ICMIC), 2017, pp. 675-679, doi: 10.1109/ICMIC.2017.8321540.

[5] S. Herdjunanto, A. P. Sandiwan, and A. I. Cahyadi, "Quadrotor proportional-derivative regulation for nonzero set point on SO(3) with disturbance compensation," in IOP Conf. Series: Materials Science and Engineering, vol. 190, 2017, pp. 1-5, doi:10.1088/1757-899X/190/1/012005.

[6] V. N. Sizykh, S. B. Antoshkin, R. A. Daneev, M. V. Bakanov, A. V. Livshits, and A. A. Aleksandrov, "Analytical design of control system mathematical models for mobile robots based on the methods of inverse problems of dynamics and modal PID controllers," in IOP Conf. Series: Materials Science and Engineering, vol. 760, pp. 1-20, 2020, doi:10.1088/1757-899X/760/1/012053.

[7] D. Kucherov, A. Kozub, and A. Rasstrygin, "Setting the PID Controller for Controlling Quadrotor Flight: a Gradient Approach," 2018 IEEE 5th International Conference on Methods and Systems of Navigation and Motion Control (MSNMC), 2018, pp. 90-93, doi: 10.1109/MSNMC.2018.8576294.

[8] M. Wasim, M. Ullah, and J. Iqbal, "Gain-scheduled proportional integral derivative control of taxi model of unmanned aerial vehicles," Rev. Roum. Sci. Techn. - Électrotechn. et Énerg., vol. 64, No. 1, pp. 75-80, 2019.

[9] U. Ahmad, M. Ahsan, A. I. Qazi, and M. A. Choudhry, "Modeling of lateral dynamics of a UAV using system identification approach," 2015 International Conference on Information and Communication Technologies (ICICT), 2015, pp. 1-5, doi: 10.1109/ICICT.2015.7469479.

[10] Z. Wang and J. Li, "3D simulation of flight control system for quad tilt rotor UAV based on flightgear," in IOP Conf. Series: Earth and Environmental Science, vol. 440, pp. 1-9, 2020, doi:10.1088/1755-1315/440/5/052056.

[11] J. Colorado, et al., "Inertial attitude control of a bat-like morphing-wing micro air vehicle," Bioinspiration \& Biomimetics, vol.8, no.1, pp. 1-25, 2013, doi:10.1088/1748-3182/8/1/019502. 
[12] O. Barabash, N. Dakhno, H. Shevchenko, and V. Sobchuk, "Integro-Differential Models of Decision Support Systems for Controlling Unmanned Aerial Vehicles on the Basis of Modified Gradient Method," 2018 IEEE 5th International Conference on Methods and Systems of Navigation and Motion Control (MSNMC), 2018, pp. 94-97, doi: 10.1109/MSNMC.2018.8576310.

[13] O. A. Mashkov, V. V. Sobchuk, O. V. Barabash, N. B. Dakhno, H. V. Shevchenko, and T. V. Maisak, "Improvement of variational-gradient method in dynamical systems of automated control for integro-differential models," Mathematical modeling and computing, vol. 6, no. 2, pp. 344-357, 2019, doi:10.23939/mmc2019.02.344.

[14] O. Barabash, N. Dakhno, H. Shevchenko, and V. Sobchuk, "Unmanned Aerial Vehicles Flight Trajectory Optimisation on the Basis of Variational Enequality Algorithm and Projection Method," 2019 IEEE 5th International Conference Actual Problems of Unmanned Aerial Vehicles Developments (APUAVD), 2019, pp. 136-139, doi: 10.1109/APUAVD47061.2019.8943869.

[15] R. Vazquez and M. Krstic, "Taking a step back: a brief history of PDE backstepping," in 20th World Congress The International Federation of Automatic Control, 2017, pp. 4258-4262.

[16] F. Alyoussef and I. Kaya, "A review on nonlinear control approaches: sliding mode control, back-stepping control and feedback linearization control," in International Engineering and Natural Sciences Conference, 2019, pp. $608-619$.

[17] M. Nasr, et al., "A comparitive study on the control of UAVs for Trajectory tracking by MPC, SMC, Backstepping, and Fuzzy Logic controllers," 2018 IEEE International Conference on Vehicular Electronics and Safety (ICVES), 2018, pp. 1-6, doi: 10.1109/ICVES.2018.8519511.

[18] A. Basri, K. A. Danapalasingam, and A. R. Husain, "Design and optimization of backstepping controller for an underactuated autonomous quadrotor unmanned aerial vehicle," Transactions of FAMENA, vol. 38, no. 3, pp. 27-44, 2014.

[19] S. Hassan, B. Abdelmajid, Z. Mourad, S. Aicha, and B. Abdenaceur, "PSO-Backstepping controller of a grid connected DFIG based wind turbine," International Journal of Electrical and Computer Engineering (IJECE), vol. 10, no. 1, pp. 856-867, 2020, doi: 10.11591/ijece.v10i1.

[20] N. Vafamand, "Adaptive Robust Neural-Network-Based Backstepping Control of Tethered Satellites With Additive Stochastic Noise," in IEEE Transactions on Aerospace and Electronic Systems, vol. 56, no. 5, pp. 3922-3930, 2020, doi: 10.1109/TAES.2020.2985276

[21] A. Giuseppi, A. Pietrabissa, S. Cilione, and L. Galvagni "Feedback linearization-based satellite attitude control with a life-support device without communications," Control Engineering Practice, vol. 90, pp. 221-230, 2019, doi: 10.1016/j.conengprac.2019.06.020.

[22] L. Fan, X. Liu, and G. Cai, "Attitude tracking and vibration control of membrane antenna satellite," Journal of the Franklin Institute, vol. 357, no. 15, pp. 10584-10599, 2020, doi: 10.1016/j.jfranklin.2020.08.034

[23] Y. Kartal, K. Subbarao, N. R. Gans, A. Dogan, and F. Lewis, "Distributed backstepping based control of multiple UAV formation flight subject to time delays," IET Digital Library, vol. 14, no. 12, pp. 1628-1638, 2020, doi: 10.1049/iet-cta.2019.1151.

[24] Y. Zahraoui, M. Akherraz, C. Fahassa, and S. Elbadaoui, "Induction motor harmonic reduction using space vector modulation algorithm", Bulletin of Electrical Engineering and Informatics, vol. 9, no. 2, pp. 452-465, 2020 doi: 10.11591/eei.v9i2.1682.

[25] Y. Dbaghi, S. Farhat, M. Mediouni, H. Essakhi, and A. Elmoudden, "Indirect power control of DFIG based on wind turbine operating in MPPT using backstepping approach," International Journal of Electrical and Computer Engineering, vol. 11, no. 3, pp. 1951-1961, 2021, doi: 10.11591/ijece.v11i3.

[26] N. Ali, W. Alam, M. Pervaiz, J. Iqbal, "Nonlinear adaptive backstepping control of permanent magnet synchronous motor," Rev. Roum. Sci. Techn.-Électrotechn. et Énerg., vol. 66, no. 1, pp. 9-14, 2021.

[27] M. Makhad, M. Zazi, and A. Loulijat., "Nonlinear control of WECS based on PMSG for optimal power extraction," International Journal of Electrical and Computer Engineering, vol. 10, no. 3, pp. 2815-2823, 2020 doi: 10.11591/ijece.v10i3.pp2815-2823.

[28] Y. Abouelmahjoub and M. Moutchou, "Nonlinear control strategy of single-phase unified power flow controller," International Journal of Electrical and Computer Engineering, vol. 11, no. 4, pp. 2864-2875, 2021 doi: 10.11591/ijece.v11i4.pp2864-2875.

[29] A. H. Mary, A. H. Miry, and M. H. Miry, "System uncertainties estimation based adaptive robust backstepping control for DC DC buck converter," International Journal of Electrical and Computer Engineering, vol. 11, no. 1, pp. 347-355, 2021, doi: 10.11591/ijece.v11i1.pp347-355.

[30] M. Madark, A. Ba-razzouk, and M. El Malah, "Linear and nonlinear controllers of a solar photovoltaic water pumping system," Bulletin of Electrical Engineering and Informatics, vol. 9, no. 5, 2020, pp. 1861-1872, doi: 10.11591/eei.v9i5.1778

[31] M. Moutchou and A. Jbari, "Fast photovoltaic IncCond-MPPT and backstepping control, using DC-DC boost converter," International Journal of Electrical and Computer Engineering, vol. 10, no. 1, pp. 1101-1112, 2020, doi: 10.11591/ijece.v10i1.pp1101-1112.

[32] M. Madark, A. Ba-razzouk, E. Abdelmounim, and M. El Malah, "Adaptive backstepping control of induction motor powered by photovoltaic generator," International Journal of Electrical and Computer Engineering, vol. 11, no. 4, pp. 2842-2855, 2021, doi: 10.11591/ijece.v11i4.pp2842-2855.

[33] D. Kucherov and A. Kozub, "Backstepping algorithm for controlling of quadrotor," in IEEE 6th International Conference Methods and Systems of Navigation and Motion Control, pp. 51-55, 2020, doi: 10.1109/MSNMC50359.2020.9255518. 
[34] M. A. M. Basri and A. Noordin, "Optimal backstepping control of quadrotor UAV using gravitational search optimization algorithm, ” Bulletin of Electrical Engineering and Informatics, vol. 9, no. 5, pp. 1819-1826, 2020, doi: 10.11591/eei.v9i5.2159

[35] I. Horowits, Synthesis of feedback systems, New York, USA: Academic Press, 2013.

[36] G.A. Korn and T.M. Korn, Mathematical handbook for Scientists and Engineers: Definitions, Theorems, and Formulas for Reference and Review, New York, USA: Dover Publications, 2000.

[37] O. Mechali, et al., "Robust finite-time trajectory tracking control of quadrotoraircraft via terminal sliding modebased active antidisturbance approach: a PIL experiment," International Journal of Aerospace Engineering, vol. 2021, pp. 1-28, 2021, doi: 10.1155/2021/5522379.

\section{BIOGRAPHIES OF AUTHORS}
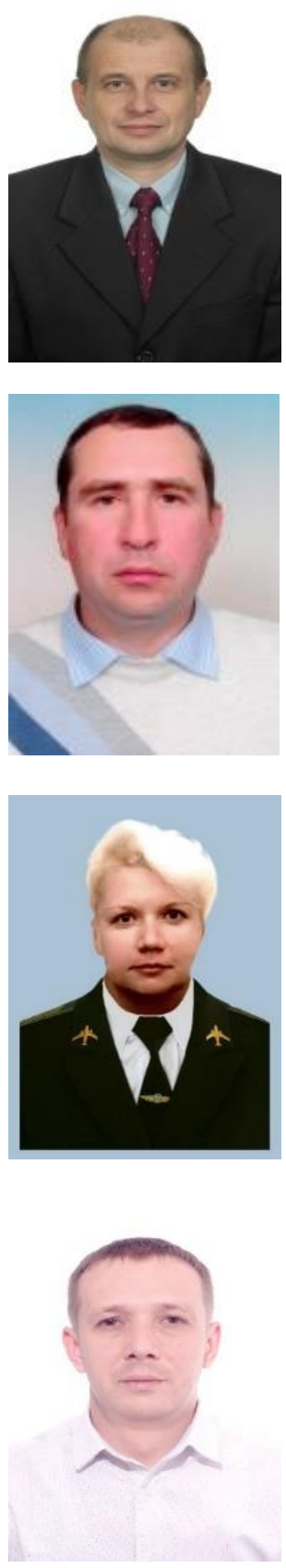

Dmytro Kucherov graduated from the Kyiv Higher Military Engineering Radiotechnical School of Air Defence Troops, Ukraine, in 1986. He received a Ph.D. degree in electrical engineering from Kyiv Higher Military Radiotechnical School of Air Defence Troops in 1995. He received the degree Doctor of Technical Science in 2009 from the Central Research Institute of Armament Armed Forces of Ukraine. Since 2012 he has been a Professor of the Department of Computerized Control Systems at National Aviation University, Kyiv, Ukraine. He is the co-author of the textbook on "Method synthesis of adaptive terminal control system". His current teaching and research interests are in the area of adaptive control, pattern recognizing in automatic control, and image processing.

Andrei Kozub graduated from the Kharkiv Nuclear Missile College, Ukraine, in 1988, received a specialty in the software of automated control systems, and a qualification engineer and mathematician. Completed postgraduate studies at the Kyiv Institute of the Air Force in 2001, $\mathrm{Ph} . \mathrm{D}$. in 2002; thesis is devoted to technical issues of building guidance system of aircraft guided missiles. Since 2003 he received the academic rank of a senior researcher and graduated from the National Defence Academy of Ukraine in 2004. Since 2012 Associate Professor in the area of uses of space systems and GIS software at the National University of Defence of Ukraine. He is a co-author of two textbooks on remote sensing of the Earth and spacecraft control of dual-use. His current teaching and research interests are in the area of space systems, devices of optoelectronic surveillance, aerospace monitoring, optimizing, complexation, efficiency, geoinformatics, pattern recognition, remote sensing.

Olha Sushchenko has been graduated from the Kyiv Polytechnic Institute, Ukraine, in 1980. She received a Ph.D. degree in military engineering from Scientific and Research Institute Hydro-device in 1991. She received the degree Doctor of Technical Science on automation of design works in 2015 from the National Aviation University, Ukraine. Since 2008 she has been a Professor of the Department of Aerospace Control Systems at the National Aviation University, Kyiv, Ukraine. She received the certificate of a full professor in 2019. She is a member of the Transport Academy of Ukraine. She is the co-author of two monographs in the area of designing aviation systems. Her current teaching and research interests are in the area of robust control and navigation systems of aerospace moving vehicles.

Ruslan Skrynkovskyy graduated from Lviv Polytechnic National University, Ukraine, in 2005. He received a Ph.D. degree in economics from Lviv University of Business and Law in 2014. Since 2020 he has been a Professor of the Department of Business Economy and Information Technology. He is a member of the Ukrainian Academy of Sciences. He is the author and co-author of more than 100 scientific papers, as well as textbooks in informatics. 